\title{
Exploring Three-Nucleon Forces via Three- and Four-Nucleon Scattering
}

\author{
Kimiko Sekiguchi ${ }^{\star}$ \\ Department of Physics, Tohoku University, Sendai, 980-8578, JAPAN \\ * kimiko@lambda.phys.tohoku.ac.jp
}

December 11, 2019

Proceedings for the 24th edition of European Few Body Conference, Surrey, UK, 2-4 September 2019

\begin{abstract}
Three-nucleon force effects in three- and four-nucleon scattering are discussed from an experimental point of view.
\end{abstract}

\section{Contents}

$\begin{array}{llr}1 & \text { Introduction } & 1\end{array}$

2 Experiments and Results of elastic $d p$ scattering at RIKEN 2

3 Experiments and Results of elastic $p+{ }^{3} \mathrm{He}$ scattering 4

4 Summary and Outlook $\quad 5$

$\begin{array}{ll}\text { References } & 7\end{array}$

\section{Introduction}

One of the main interest in nuclear physics is to understand the forces acting between nuclear constituents. A hot topic in the study of nuclear forces is to clarify the roles of three-nucleon forces (3NFs) that appear when more than two nucleons $(A \geq 3)$ interact, and to describe various phenomena of nuclei by explicitly taking into account nucleon-nucleon (NN) interactions combined with 3NFs. The 3NFs arise naturally in the standard meson-exchange picture [1] as well as in the framework of chiral effective field theory ( $\chi$ EFT) which has a link to QCD $[2,3]$.

Few-nucleon scattering offers a good opportunity to study dynamical aspects of 3NFs, which are momentum, spin and isospin dependent. It provides not only cross sections but also a variety of spin observables at different incident nucleon energies. Direct comparison between the experimental data and the rigorous numerical calculations in terms of Faddeev 
theory based on the realistic bare nuclear potentials provides information on 3NFs. An indication of $3 \mathrm{NF}$ in few-nucleon scattering was first pointed out in the cross section minima for nucleon-deuteron $(N d)$ elastic scattering at intermediate energies $(E / A \gtrsim 60 \mathrm{MeV})[4]$. Since then experimental studies of higher-energy proton-deuteron $(p d)$ and neutron-deuteron $(n d)$ elastic scattering covering incident energies of up to $\sim 300 \mathrm{MeV}$ have been performed at the facilities, e.g. RIKEN, RCNP, KVI, and IUCF, providing precise data of the cross sections as well as various types of the spin observables [5]. Compilations of recent experiments for $p d$ and nd elastic scattering at intermediate energies are shown in Fig.1.

The four-nucleon ( $4 N$ ) systems could also play an important role for the study of 3NFs. $3 \mathrm{NF}$ effects are expected to be sizable in the $4 \mathrm{~N}$ system. In addition, tests of the iso-spin $T=3 / 2$ channel in any $3 \mathrm{NFs}$ can be performed in a $4 N$ system such as proton- ${ }^{3} \mathrm{He}$ scattering, while the $N d$ scattering is essentially a pure isospin $T=1 / 2$ state. In recent years, remarkable theoretical work in solving the $4 \mathrm{~N}$ scattering problem with realistic Hamiltonian has been reported even above four-nucleon breakup threshold energies [8-10], which opens up new possibilities to study properties of $3 \mathrm{NFs}$.

With the aim of pinning down detailed properties of the 3NFs, experimental programs of deuteron-proton $(d p)$ scattering as well as proton $-{ }^{3} \mathrm{He}\left(p+{ }^{3} \mathrm{He}\right)$ scattering are ongoing at RIKEN, RCNP Osaka University, and CYRIC Tohoku University, in Japan. In this contribution we introduce recently conducted experiments and present the results of comparison between the experimental data and the theoretical predictions based on the realistic bare nuclear potentials.

\section{Experiments and Results of elastic $d p$ scattering at RIKEN}

The experiments of $d p$ elastic scattering at RIKEN Accelerator Facility was conduced using vector and tensor polarized deuteron beams at 70, 100, and $135 \mathrm{MeV} /$ nucleon [11] in conjunction with the magnetic spectrometer SMART [12]. Measured observables are the cross section, all deuteron analyzing powers $\left(i T_{11}, T_{20}, T_{21}\right.$, and $\left.T_{22}\right)$, and deuteron to proton polarization transfer coefficients $\left(K_{y}^{y^{\prime}}, K_{y y}^{y^{\prime}}, K_{x x}^{y^{\prime}}\right.$, and $\left.K_{x z}^{y^{\prime}}\right)$ [13]. Later, the experiments were extended to the RIKEN RI Beam Factory (RIBF). All deuteron analyzing powers were obtained at $190,250,294 \mathrm{MeV} /$ nucleon $[14,15]$.

In Fig. 2 some representative results reported in Refs. [13-15] are shown in Fig. 2. The experimental data are compared with the Faddeev calculations with and w/o 3NFs. The red (blue) bands are the calculations with (without) Tucson-Melbourne99 (TM99) 3NF [16] based on the modern NN potentials, i.e. CD Bonn [17], AV18 [18], Nijmegen I and II [19]. The solid lines are the calculations based on the AV18 potential with including the Urbana IX 3NF [20]. The $3 \mathrm{NFs}$ considered here are $2 \pi$-exchange types. For most of the observables shown in the figure large differences are found between the data and the calculations based on NN forces only at the backward angles. These discrepancies become larger with increasing an incident energy. For the cross section the 3NFs remove the discrepancies at lower energies. At higher energies the differences still remain even including the 3NF potentials at the angles $\theta_{\text {c.m. }} \gtrsim 120^{\circ}$, which lie to the very backward angles $\theta_{\text {c.m. }} \sim 180^{\circ}$ at $250 \mathrm{MeV} /$ nucleon. For the vector analyzing power $i T_{11}$ the results of comparison are quite similar to those for the cross section. However for the tensor analyzing power $T_{22}$ the different features are found. The calculations taking into account the 3NFs do not explain at the lower two energies.

The results of comparison between the data and the calculations based on the above phenomenological nuclear potentials have been pushing us into more detailed study of threenucleon scattering based on the $\chi$ EFT nuclear potential. Figure 4 shows the recent calculations based on the locally regularized (regulator $R=0.9 \mathrm{fm}$ ) $\mathrm{N}^{4} \mathrm{LO} \chi$ EFT NN potential [21]. 
$p d$ and $n d$ Elastic Scattering at $65-400 \mathrm{MeV} /$ nucleon

\begin{tabular}{|c|c|c|c|c|}
\hline Observable & 100 & 200 & 300 & 400 \\
\hline$\frac{d \sigma}{d \Omega}$ & - & ค००८ ๑ ० & & $\dot{\varphi}$ \\
\hline $\begin{array}{ll}\vec{p} & A_{y}{ }^{p} \\
\vec{n} & A_{y}{ }^{n}\end{array}$ & & לים & $?$ & $\dot{\varphi}$ \\
\hline $\begin{array}{cc}\vec{d} \quad & i T_{11} \\
& T_{20} \\
& T_{22} \\
& T_{21}\end{array}$ & "ִ & $\begin{array}{lll}0 & 0 \\
0 & 0 \\
0 & 0 \\
0 & 0 \\
0 & 0\end{array}$ & $\begin{array}{ll}0 & 0 \\
- & \vdots \\
\bullet & \vdots \\
\bullet & \vdots\end{array}$ & $\dot{\varphi}$ \\
\hline $\begin{array}{cc}\vec{p} \rightarrow \vec{p} & K_{y}^{y^{\prime}} \\
& K_{x}^{x^{\prime}} \\
& K_{x}^{z^{\prime}} \\
& K_{z}^{x^{\prime}} \\
& K_{z}^{z^{\prime}}\end{array}$ & & $\pi$ threshold: & $\begin{array}{l}\bullet \\
\bullet \\
\bullet \\
\bullet \\
\bullet \\
\bullet\end{array}$ & \\
\hline $\begin{aligned} \vec{d} \rightarrow \vec{p} & K_{y}^{y^{\prime}} \\
& K_{x x}^{y^{\prime}} \\
& K_{y y}^{y^{\prime}} \\
& K_{x z}^{y^{\prime}}\end{aligned}$ & $\bullet$ & $\begin{array}{l}\bullet \\
\bullet \\
\bullet \\
\bullet\end{array}$ & & \\
\hline$\vec{p} \rightarrow \vec{a} \quad K_{y}^{y^{\prime}}$ & & & & $\dot{\vdots}$ \\
\hline $\begin{array}{ll}\vec{p} \vec{d} & C_{i, j} \\
& C_{i j, \mathrm{k}}\end{array}$ & & - $\quad \dot{0}$ & & \\
\hline
\end{tabular}

figure

figure caption is below the

Figure 1: Compilations of recent experiments of $p d$ and nd elastic scattering at 65$400 \mathrm{MeV} /$ nucleon. Solid circles denote $p d$ experiments and open circles denote $n d$ experiments. The measurements with large circles cover the wide angular range, while those with small circles cover the limited angular range. 
The $\mathrm{N}^{4} \mathrm{LO}$ chiral potential predictions are close to those based on the semi-phenomenological NN potentials shown in Fig. 2. In order to see how $\chi$ EFT 3NFs describe the data, theoretical treatments are now in progress [22].
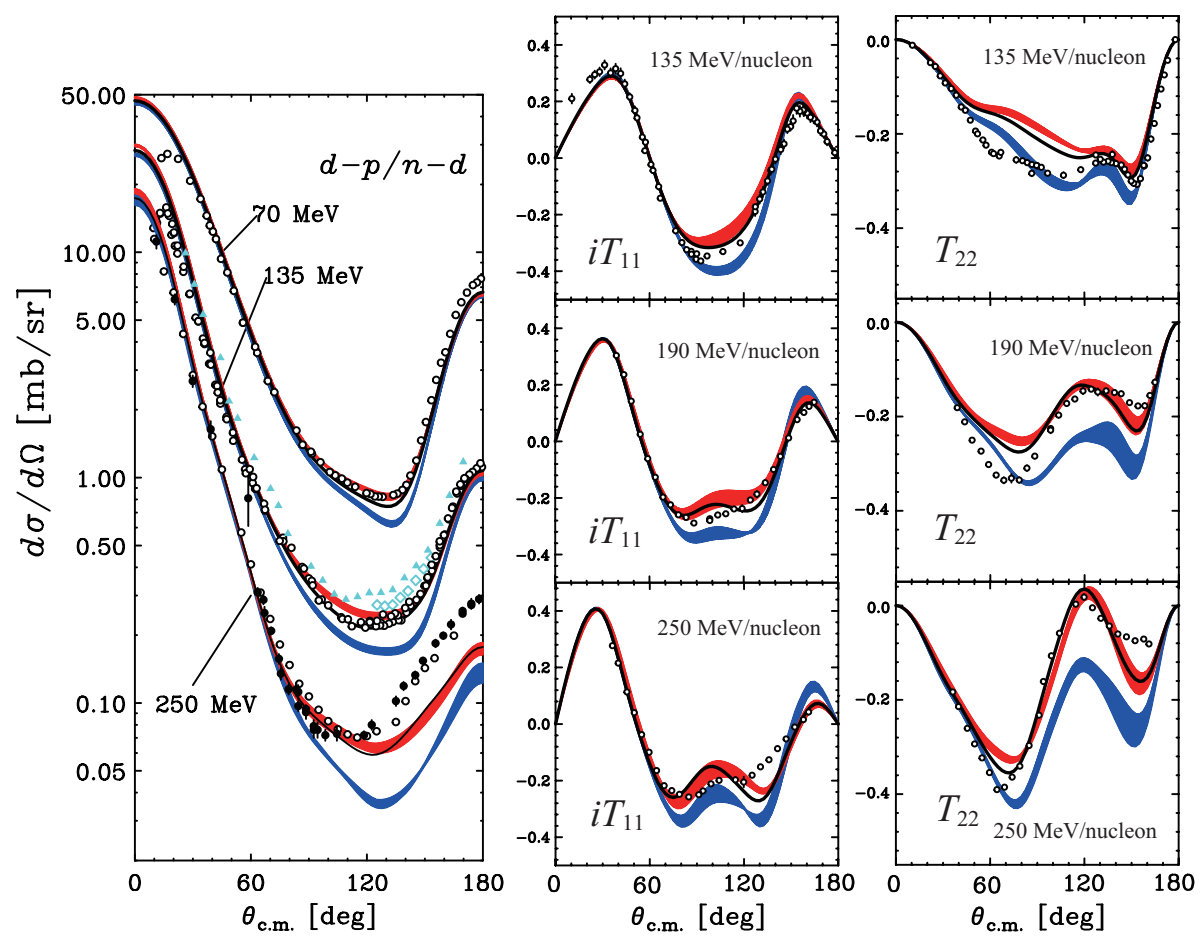

Figure 2: Differential cross section and deuteron analyzing powers $i T_{11}, T_{22}$ for elastic $d p$ scattering. Data shown in blue are taken from Refs. [23,24]. See text for descriptions for the theoretical calculations.

\section{Experiments and Results of elastic $p+{ }^{3} \mathrm{He}$ scattering}

Following the experiments of $d p$ scattering we proceed to the experiments of the $p+{ }^{3} \mathrm{He}$ scattering at incident nucleon energies above $\sim 65 \mathrm{MeV}$. The experiments were conducted at the two cyclotron facilities, RCNP, Osaka University, and CYRIC, Tohoku University. The measured observables are the cross section, the proton analyzing power $A_{y}(p)$, the ${ }^{3} \mathrm{He}$ analyzing power $A_{y}\left({ }^{3} \mathrm{He}\right)$ as well as the spin correlation coefficient $C_{y, y}$. Detailed descriptions of the experiments are reported in Refs. [25].

In Fig. 4 parts of the data are are shown in Fig. 4. In the conference the data were compared with rigorous numerical four-nucleon calculations based on the Alt, Grassberger, and Sandhas equation and modern NN potentials (CD Bonn [17], AV18 [18], INOY04 [27], and SMS51 and SMS53 (semilocal momentum-space regularized chiral $N N$ potentials) [26]) [28]. The calculations are not shown here. For the cross section the data are about 35\% larger than the theoretical predictions at the angles $\theta_{\text {c.m. }}=80^{\circ}-140^{\circ}$ where the cross section takes minimum. It should be noted that the difference found in the cross section is about twice large as that for the $p d$ elastic scattering at $65 \mathrm{MeV}$ [4]. As for the spin observables, the ${ }^{3} \mathrm{He}$ analyzing power $A_{y}\left({ }^{3} \mathrm{He}\right)$ as well as the spin correlation coefficient $C_{y, y}$ large differences are found between the data and the calculations at the backward angles $\theta_{\text {c.m. }} \geq 80^{\circ}$, while the proton analyzing power $A_{y}(p)$ data are well reproduced by the theoretical predictions. The data were also compared with the calculations with the $\Delta$-isobar excitations combined with the CD Bonn potential. One 

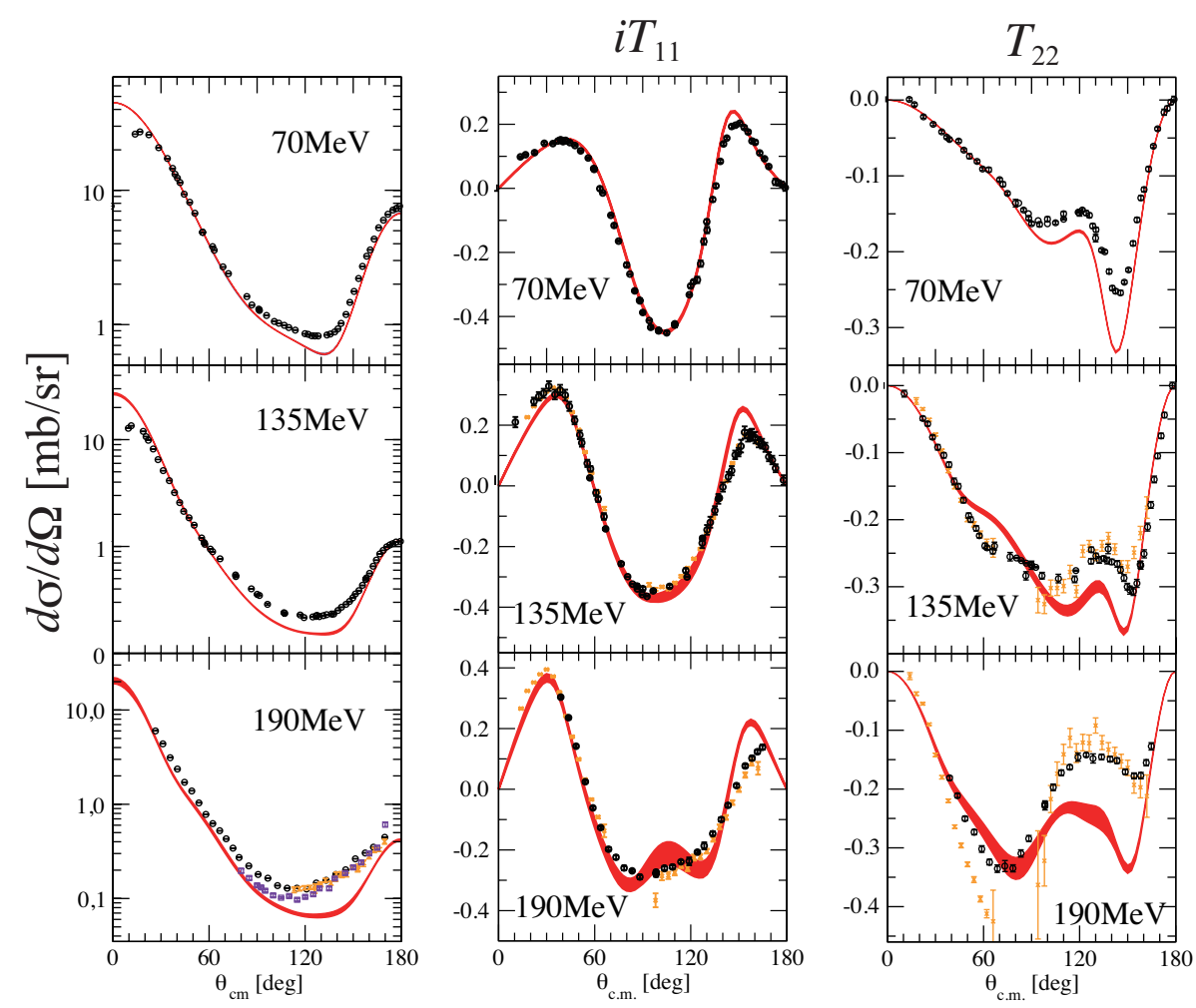

Figure 3: Differential cross section and deuteron analyzing powers $i T_{11}, T_{22}$ for elastic $d p$ scattering. The bands in the figure are the calculations based on the locally regularized (regulator $R=0.9 \mathrm{fm}$ ) $\mathrm{N}^{4} \mathrm{LO} \chi \mathrm{EFT}$ NN potential.

could infer the effects of three-nucleon forces, mainly the Fujita-Miyazawa type 3NFs. The $\Delta$-isobar effects are very small for the cross section and the ${ }^{3} \mathrm{He}$ analyzing power $A_{y}\left({ }^{3} \mathrm{He}\right)$, and then they do not remedy the difference between the data and the calculations based on the NN potentials. Meanwhile sizable effects are found for the spin correlation coefficient $C_{y, y}$. The results of comparison indicate that $p+{ }^{3} \mathrm{He}$ elastic scattering at these energies could provide a rich source for $3 \mathrm{NFs}$, and their effects are expected to be larger than those for the $\mathrm{Nd}$ elastic scattering.

\section{Summary and Outlook}

Few-nucleon scattering provides rich sources to explore the properties of 3NFs that are momentum, spin and iso-spin dependent. To study the detailed properties of the 3NFs, experimental programs of deuteron-proton $(d p)$ scattering as well as proton $-{ }^{3} \mathrm{He}\left(p+{ }^{3} \mathrm{He}\right)$ scattering using the polarized beam and target systems are ongoing at RIKEN, RCNP Osaka University, and CYRIC Tohoku University, in Japan.

In this contribution, the experimental results obtained with polarized deuteron beams at RIKEN are presented. The data for the $d p$ elastic scattering are compared with the rigorous numerical calculations based on the phenomenological NN potentials combined with the two$\pi$ exchange 3NFs as well as the $\chi$ EFT NN potential. The energy and angular dependent results of the cross sections as well as the deuteron analyzing powers show that one needs to take into account chiral 3NFs in future calculations.

The 3NF effects could also be sizable in the four-nucleon scattering systems in which tests of the isospin $T=3 / 2$ channel in $3 \mathrm{NFs}$ can be performed. As the first step we conducted 
$p+{ }^{3} \mathrm{He}$ Elastic Scattering
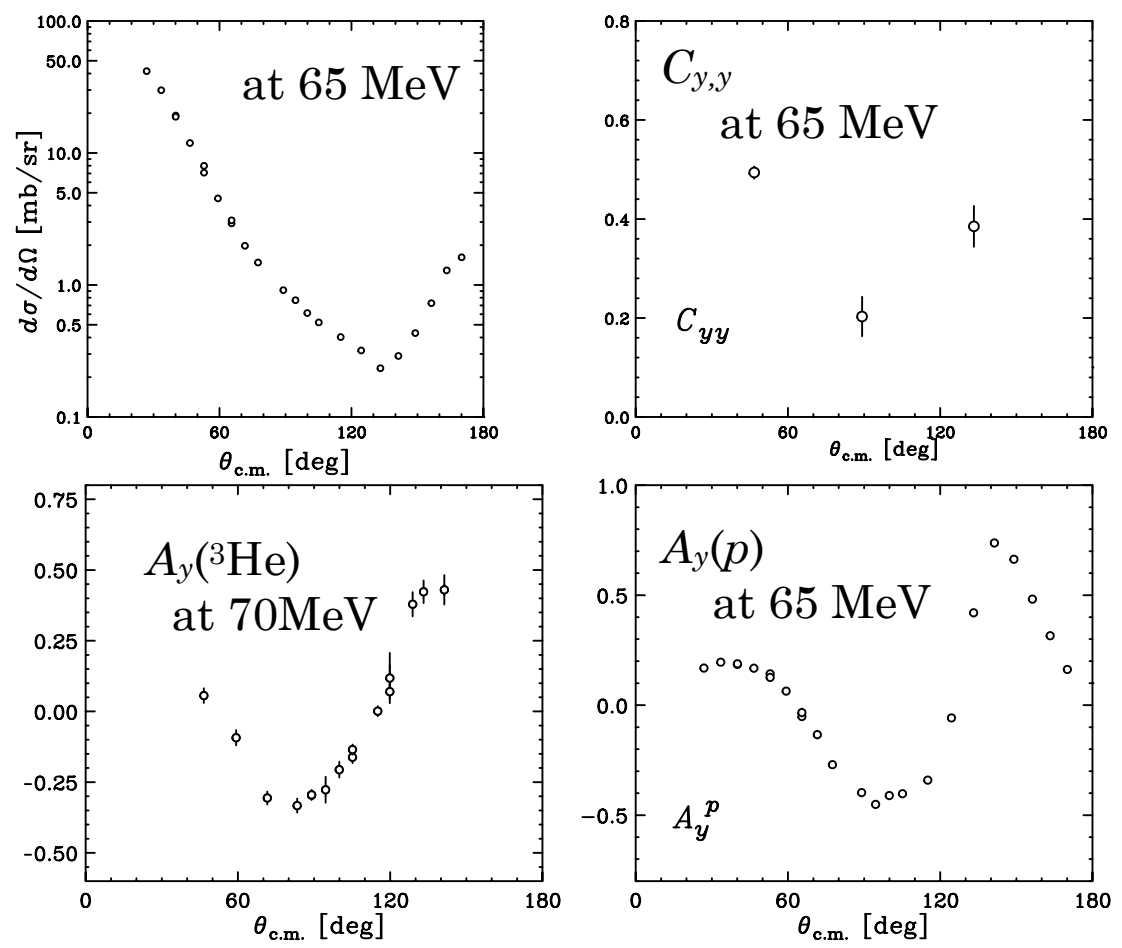

Figure 4: Cross section, proton analyzing power $A_{y}(p),{ }^{3} \mathrm{He}$ analyzing power $A_{y}\left({ }^{3} \mathrm{He}\right)$, and spin correlation coefficient $C_{y, y}$ for $p+{ }^{3} \mathrm{He}$ elastic scattering.

experiments of $p+{ }^{3} \mathrm{He}$ elastic scattering at around $65 \mathrm{MeV}$ using the polarized proton beam and the polarized ${ }^{3} \mathrm{He}$ target. For almost the all measured observables large differences are found between the data and the theoretical calculations based on the various NN potentials at the angles around the cross minimum. These features are similar to those obtained in the $N d$ elastic scattering.

As the next step we are planning the following experiments; i) measurements of $p+{ }^{3} \mathrm{He}$ scattering at $65-200 \mathrm{MeV}$, and ii) measurements of spin-correlation coefficients for $d p$ scattering at $65-200 \mathrm{MeV}$. These data would provide a valuable source to test nuclear potentials including 3NFs and/or to determine the low-energy constants of the chiral EFT nuclear potentials. Such theoretical work is now in progress [22].

\section{Acknowledgements}

The author would like to thank the collaborators for the experiments performed at RIKEN RI Beam Factory, RCNP Osaka University, and CYRIC Tohoku University. She is also grateful to the strong supports from the theorists, H. Witała, W. Glöckle, H. Kamada, J. Golak, A. Nogga, R. Skibiński, E. Epelbaum, P. U. Sauer, A. Deltuva, and A. C. Fonseca. This work was supported financially in part by the Grants-in-Aid for Scientific Research No. 20684010, 24684013, 25105502, 16H02171, and No. 18H05404 of the Ministry of Education, Culture, Sports, Science, and Technology of Japan. 


\section{References}

[1] J. Fujita and H. Miyazawa, Pion Theory of Three-Body Forces, Prog. Theor. Phys. 17, 360 (1957), doi:doi:10.1143/PTP.17.360.

[2] U. van Kolck, Few-nucleon forces from chiral Lagrangians, Phys. Rev. C 49, 2932 (1994), doi:doi:10.1103/PhysRevC.49.2932.

[3] E. Epelbaum, H.-W. Hammer, U.-G. Meißner, Modern theory of nuclear forces, Rev. Mod. Phys. 81, 1773 (2009) doi:doi:10.1103/RevModPhys.81.1773.

[4] H. Witała et al., Cross Section Minima in Elastic Nd Scattering: Possible Evidence for Three-Nucleon Force Effects, Phys. Rev. Lett. 81, 1183 (1998), doi:doi:10.1103/PhysRevLett.81.1183.

[5] See references in N. Kalantar-Nayestanaki, E. Epelbaum, J. G. Messchendorp and A. Nogga, Signatures of three-nucleon interactions in few-nucleon systems, Rep. Prog. Phys. 75, 016301 (2012), doi:doi:10.1088/0034-4885/75/1/016301.

[6] S. C. Pieper et al., V. R. Pandharipande, R. B. Wiringa, and J. Carlson, Realistic mod- els of pion-exchange three-nucleon interactions, Phys. Rev. C. 64, 014001 (2001), doi:doi:10.1103/PhysRevC.64.014001.

[7] A. Akmal, V. R. Pandharipande and D. G. Ravenhall, Equation of state of nucleon matter and neutron star structure, Phys. Rev. C 58, 1804 (1998), doi:doi:10.1103/PhysRevC.58.1804.

[8] A. Deltuva and A. C. Fonseca, Calculation of proton $-{ }^{3}$ He elastic scattering between 7 and 35 MeV, Phys. Rev. C 87, 054002 (2013), doi:doi:10.1103/PhysRevC.87.054002.

[9] M. Viviani, L. Girlanda, A. Kievsky and L. E. Marcucci, Effect of three-nucleon interactions in p-3 He elastic scattering, Phys. Rev. Lett. 111, 172302 (2013), doi:doi:10.1103/PhysRevLett.111.172302.

[10] Rimantas Lazauskas Elastic proton scattering on tritium below the $n-{ }^{3}$ He threshold Phys. Rev. C 79, 054007 (2009), doi:doi:10.1103/PhysRevC.79.054007.

[11] H. Okamura et al., Development of the RIKEN polarized ion source AIP Conference Proceedings 293, 84 (1993), doi:doi:10.1063/1.45151.

[12] T. Ichihara et al., Spin isospin resonance observed in the $\left(d,{ }^{2} \mathrm{He}\right)$ and $\left({ }^{1} 2 C,{ }^{1} 2 N\right)$ reactions at $E / A=135 \mathrm{MeV}$ Nucl. Phys. A 569 287c-296c (1994). doi:doi:10.1016/03759474(94)90119-8

[13] For example, K. Sekiguchi et al., Complete set of precise deuteron analyzing powers at intermediate energies: Comparison with modern nuclear force predictions, Phys. Rev. C 65, 034003 (2002) doi:doi:10.1103/PhysRevC.65.034003;

K. Sekiguchi et al., Resolving the Discrepancy of $135 \mathrm{MeV}$ pd Elastic Scattering Cross Sections and Relativistic Effects, Phys. Rev. Lett 95, 162301 (2005), doi:doi:10.1103/PhysRevLett.95.162301 and see references therein.

[14] K. Sekiguchi et al. Complete set of deuteron analyzing powers for d p elastic scattering at 250-294 MeV /nucleon and the three-nucleon force, Phys. Rev. C 89, 064007 (2014), doi:doi:10.1103/PhysRevC.89.064007. 
[15] K. Sekiguchi et al., Complete set of deuteron analyzing powers from $\vec{d} p$ elastic scattering at $190 \mathrm{MeV} /$ nucleon, Phys. Rev. C 96, 064001 (2017), doi:doi:10.1103/PhysRevC.96.064001.

[16] S. A. Coon and H. K. Han, Reworking the Tucson-Melbourne three nucleon potential FewBody Syst. 30, 131 (2001), doi:doi:0.1007/s006010170022

[17] R. Machleidt, High-precision,charge-dependentbonnnucleon-nucleonpotential, Phys.Rev. C 63, 024001 (2001), doi:doi:10.1103/PhysRevC.63.024001.

[18] R. B. Wiringa, V. G. J. Stoks and R. Schiavilla, Accurate nucleon-nucleon potential with charge-independence breaking, Phys. Rev. C 51, 38 (1995), doi:doi:10.1103/PhysRevC.51.38.

[19] V. G. J. Stoks, R. A. M. Klomp, C. P. F. Terheggen and J. J. de Swart, Construction of high-quality NN potential models, Phys. Rev. C 49, 2950 (1994), doi:doi:10.1103/PhysRevC.49.2950.

[20] B. S. Pudliner, V. R. Pandharipande, J. Carlson, Steven C. Pieper, and R. B. Wiringa, Quantum Monte Carlo calculations of nuclei withA $\leq$ 7, Phys. Rev. C 56, 1720 (1997), doi:doi:10.1103/PhysRevC.56.1720.

[21] S. Binder et al., Few-nucleon systems with state-of-the-art chiral nucleon-nucleon forces Phys. Rev. C93, 044002 (2016), doi:doi:10.1103/PhysRevC.93.044002.

[22] E. Epelbaum et al., Towards high-order calculations of three-nucleon scattering in chiral effective field theory, http://arxiv.org/abs/1907.03608.

[23] K. Ermisch et al., Systematic investigation of the elastic proton-deuteron differential cross section at intermediate energies, Phys. Rev. C 68, 051001(R) (2003) doi:doi:10.1103/PhysRevC.68.051001

[24] A. Ramazani-Moghaddam-Arani et al., Elastic proton-deuteron scattering at intermediate energies, Phys. Rev. C 78, 014006 (2008) doi:doi:10.1103/PhysRevC.78.014006

[25] M. Inoue et al., Measurement of spin correlation coefficients in $p-{ }^{3} \mathrm{He}$ scattering at $65 \mathrm{MeV}$; S. Nakai et al., Measurement for $p^{-3} \mathrm{He}$ elastic scattering with a $65 \mathrm{MeV}$ polarized proton beam; A. Watanabe et al., Measurement of ${ }^{3} \mathrm{He}$ analyzing power for $p-{ }^{3} \mathrm{He}$ scattering using the polarized ${ }^{3} \mathrm{He}$ target, this conference proceedings therein.

[26] P. Reinert, H. Krebs and E. Epelbaum, Semilocal momentum-space regularized chiral two-nucleon potentials up to fifth order, Eur. Phys. J. A 54, 86 (2018), doi:doi:10.1140/epja/i2018-12516-4.

[27] P. Doleschall, Influence of the short range nonlocal nucleon-nucleon interaction on the elastic $n-d$ scattering: Below $30 \mathrm{MeV}$, Phys. Rev. C 69, 054001 (2004), doi:doi:10.1103/PhysRevC.69.054001.

[28] A. Deltuva, private communications. 